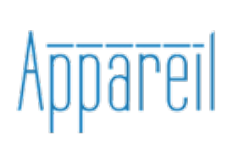

Appareil

2| 2008

Autour de Simondon

\title{
Du mort qui saisit le vif
}

Sur l'actualité de l'ontologie simondonienne

Jean-Hugues Barthélémy

\section{(2) OpenEdition}

Journals

Édition électronique

URL : http://journals.openedition.org/appareil/599

DOI : 10.4000/appareil.599

ISSN : 2101-0714

Éditeur

MSH Paris Nord

Référence électronique

Jean-Hugues Barthélémy, « Du mort qui saisit le vif », Appareil [En ligne], 2 | 2008, mis en ligne le 17 septembre 2008, consulté le 30 juillet 2020. URL : http://journals.openedition.org/appareil/599; DOI : https://doi.org/10.4000/appareil.599

Ce document a été généré automatiquement le 30 juillet 2020.

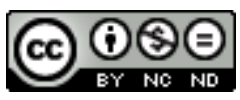

Appareil est mis à disposition selon les termes de la Licence Creative Commons Attribution - Pas d'Utilisation Commerciale - Pas de Modification 4.0 International. 


\section{Du mort qui saisit le vif}

Sur l'actualité de l'ontologie simondonienne

Jean-Hugues Barthélémy

\section{NOTE DE L'ÉDITEUR}

Ce texte est celui d'une conférence donnée à Paris le 16 juin 2007, dans le cadre du colloque « Actualité de Simondon » organisé par le Centre Georges Canguilhem de l'université de Paris 7 - Denis Diderot et le Collège international de philosophie.

\section{Introduction : le chimique, l'apoptose et l'artefact, ou I'hypothèse des trois types de " non-vie » qui conditionnent la vie comme évolution}

1 Ainsi que l'indique le titre de mon Introduction, je ne m'interrogerai pas, si ce n'est peut-être dans ma Conclusion, sur la distinction pour ainsi dire métaphysique entre «le» mort et «la » mort. Ce qui m'intéresse est plus généralement la présence de la "non-vie » dans la vie, et comme condition même de la vie. Je voudrais suggérer qu'aux différents stades de la vie en tant qu'évolution correspondent différents types de nonvie essentielle. Le « non-vivant » peut certes désigner l'artefact, mais il signifie d'abord l'inerte naturel, donc le physique. Simondon, lui, voit dans le physique et le «vital», comme il dit, deux « régimes d'individuation ». Mais en choisissant de traiter du «mort qui saisit le vif» je me propose en fait, ainsi qu'il apparaîtra, de couvrir toute l'ontologie génétique de L'individuation à la lumière des notions de forme et d'information ${ }^{1}$ en tant qu'elle fait dériver du vivant le troisième régime d'individuation lui-même, dit par Simondon «transindividuel ». Or, le fil directeur de cette traversée de l'ontologie génétique est d'autre part ce qui à mon sens permet à la fois d'unifier celle-ci et de la dépasser, parce que ce fil directeur est ce que j'ai nommé ailleurs le «sens autotranscendant ${ }^{2}$ " de l'ontologie génétique simondonienne. Tel sera l'enjeu de mon propos, parce que l'exégèse de l'œuvre simondonienne à laquelle ont procédé mes deux 
premiers ouvrages se voulait déjà polémique dans sa fidélité même, et soucieuse par là de trouver chez Simondon ce qui pouvait lui donner toute son actualité.

2 L'expression « du mort qui saisit le vif » est issue de Marx, qui écrivait en effet au seuil du Capital : « Nous avons à souffrir non seulement de la part des vivants, mais encore de la part des morts. Le mort saisit le vif ! ${ }^{3}$ " Marx concluait ici une remarque sur les conséquences politiques et sociales «à contre-temps ${ }^{4}$ » qu'engendrent certains modes passés de production. Je voudrais donner pour ma part un autre sens à sa formule, plus large et plus proche du célèbre propos d'Auguste Comte sur l'historicité de l'humanité comme présence des morts. Ma thèse sera plus précisément la suivante : l'humanité est cette forme de vie psycho-sociale qui, par le biais des artefacts non-vivants qui lui servent de support et qui fondent son historicité, prolonge la vie animale bio-psychique dont la condition non-vivante n'est pas encore l'artefact mais la simple apoptose («suicide cellulaire »), et dont l'origine est une troisième forme de «non-vie » : le non-vivant chimique.

Pour qu'il n'y ait pas de malentendu sur cette thèse, je précise d'emblée, et en remontant l'ordre de ses différents points, que :

4 1. il est admis ici que la vie du vivant vient de ce qui n'est pas elle. Simondon lui-même, tout en refusant le mécanisme en tant que réductionnisme appliqué à la vie, admet que le vitalisme n'est pas non plus défendable. Sa manière propre de refuser le mécanisme consiste alors à penser le physique et le vital comme venant tous deux d'une réalité "pré-physique et pré-vitale», parce que préindividuelle. Par ailleurs Simondon envisage d'appliquer l'idée de néoténie au passage du non-vivant vers le vivant: l'individuation vitale serait la perpétuation d'une phase inchoative de l'individuation physique elle-même. Je n'aurai pas l'occasion de revenir ici sur cette question de l'origine non-vivante de la vie, et me consacrerai plutôt à la question de l'apoptose comme deuxième forme de «non-vie » rendant possible la vie. J'analyserai le texte de Simondon qui exprime une intuition allant dans le sens de cette réalité récemment confirmée et admise par la biologie, après un siècle de questionnements isolés.

5 2. les artefacts produits par les animaux autres que l'homme, comme par exemple le nid de l'oiseau ou la ruche des abeilles, ne visent pas à rendre possible une vie psychosociale, mais seulement une vie bio-psychique ou une vie bio-sociale: comme le remarquait Simondon, le «social pur», qu'il faut entendre par opposition au psychosocial et non par opposition au biologique, existe chez les insectes parce que leur caractère social ne vient pas nourrir un psychisme. Réciproquement les oiseaux et plus encore les mammifères développent un psychisme mais sans passer par le social. Seuls les primates et plus encore l'homme sont du psycho-social, c'est-à-dire une réalité dans laquelle le psychisme individuel se développe paradoxalement à partir du collectif. Ce paradoxe est celui de ce que l'on nomme l'« intériorité » ou plutôt, avec Simondon, la "personnalité », dont Simondon disait qu'elle ne pouvait justement être pensée à partir de l'opposition extérieur/intérieur (ou transcendance/immanence).

6 3. Lorsque j'affirme que les artefacts rendent possible une vie psycho-sociale et que celle-ci ne se réalise pleinement que chez l'homme, je ne pose pas le langage à côté des artefacts, ni n'oublie d'autre part les artefacts produits par nos «cousins psychosociaux " les primates. Car d'une part le langage est lui aussi un artefact, sans doute d'ailleurs indispensable pour que les autres artefacts puissent devenir des supports de notre personnalité psycho-sociale. Par le biais du langage, où s'élabore la pensée, les artefacts produits dans le «monde extérieur» nourrissent en retour l'esprit humain. 
Cette thèse correspond en fait au prolongement par Bernard Stiegler de travaux qui étaient déjà une grande source pour Simondon : je veux parler des travaux de LeroiGourhan sur le parallélisme entre le développement du langage et celui des outils. D'autre part les artefacts produits par les primates ne sont pas conservés par eux après utilisation, et ne peuvent ainsi définir un monde historique venant nourrir les esprits, même si ces artefacts sont bien un prolongement du corps vivant.

\section{L'artefact, ou la « non-vie » qui rend possible une vie psycho-sociale}

7 Je remonterai l'ordre de la complexification en sens inverse et commencerai par la question du régime transindividuel d'individuation en tant qu'il est une vie psychosociale conditionnée par cette forme ultime de "non-vie » qu'est l'artefact. Qu'il s'agisse là d'une question, y compris lorsque l'on part de Simondon, tient au fait que la vie psycho-sociale et la culture semblent devoir posséder comme "phase» la technique, ainsi que l'avait magistralement montré Simondon dans Du mode d'existence des objets techniques $^{5}$. Or la question est de savoir si cette phase nécessaire ne serait pas encore davantage, c'est-à-dire un fondement et un cadre pour les autres phases de la culture. On sait que chez Simondon la technique n'est qu'une phase issue du "déphasage » de l'« unité magique primitive », laquelle ne semble pas contenir à ses yeux la présence d'artefacts mais seulement celle de "points clés » naturels. C'est d'ailleurs ce que j'ai reproché à Simondon au terme du second volet de mon exégèse polémique ${ }^{6}$. Mais je voudrais revenir ici sur les éléments de sa pensée, et peut-être aussi les tensions en elle, qui peuvent appeler eux-mêmes à un remaniement de cette pensée en direction d'une fondation du transindividuel ou du psycho-social sur les artefacts. .

Dans sa Thèse complémentaire pour le Doctorat d'État Du mode d'existence des objets techniques, Simondon revient sur la question du transindividuel qu'il avait traitée dans la Thèse principale L'individuation à la lumière des notions de forme et d'information. Il écrit :

l'objet technique pris selon son essence, c'est-à-dire l'objet technique en tant qu'il a été inventé, pensé et voulu, assumé par un sujet humain, devient le support et le symbole de cette relation que nous voudrions nommer transindividuelle. [...] Par l'intermédiaire de l'objet technique se crée alors une relation interhumaine qui est le modèle de la transindividualité. On peut entendre par là une relation qui ne met pas les individus en rapport au moyen de leur individualité constituée les séparant les uns des autres, ni au moyen de ce qu'il y a d'identique en tout sujet humain, par exemple les formes a priori de la sensibilité, mais au moyen de cette charge de réalité préindividuelle, de cette charge de nature qui est conservée avec l'être individuel, et qui contient potentiels et virtualité. L'objet qui sort de l'invention technique emporte avec lui quelque chose de l'être qui l'a produit, exprime de cet être ce qui est le moins attaché à un hic et nunc; on pourrait dire qu'il y a de la nature humaine dans l'être technique, au sens où le mot de nature pourrait être employé pour désigner ce qui reste d'originel, d'antérieur même à l'humanité constituée en l'homme ${ }^{7}$.

Pourquoi la pensée du transindividuel est-elle ici reprise par le biais d'une pensée de la technique pourtant absente, elle, de la Thèse principale? Avant de répondre, deux remarques préalables doivent être faites sur la lettre même du texte. Premièrement, ce que dit la fin de ce passage sur le sens du mot «nature " permet de comprendre qu'en faisant de l'objet technique le «support» de la relation transindividuelle, le début du 
passage n'entendait pas détacher l'homme de «la nature » et contredire la Thèse principale. C'est bien plutôt parce que l'objet technique est érigé au statut de support de la relation transindividuelle que la pensée simondonienne échappe à ce qu'elle combat sous le nom d'" anthropologie» essentialiste. En effet l'objet technique est pour Simondon nature dans l'homme - et non pas « nature » humaine ou essence de l'homme : «l'homme invente en mettant en œuvre son propre support naturel, cet apeiron qui reste attaché à chaque être individuel ${ }^{8} »$. C'est donc en subvertissant l'opposition nature/technique que Simondon entend ici subvertir l'opposition nature/humanité, tout comme l'Introduction de l'ouvrage annonçait une subversion de cette troisième opposition qu'est l'opposition humanisme/technicisme'.

Deuxièmement, le passage cité est lui-même habité par une tension, puisqu'il fait de l'objet technique à la fois le «support » et le « symbole » de la transindividualité, ce qui ne revient pas tout à fait au même. Pourtant c'est l'idée de symbole qui prévaut dans l'ouvrage, la «relation interhumaine» qui se fait "par l'intermédiaire de l'objet technique " étant par ailleurs, elle, le "modèle de la transindividualité", disait Simondon. Le propos de $\mathrm{Du}$ mode d'existence des objets techniques consiste plus généralement à faire de l'objet technique un paradigme pour la compréhension de ce que Simondon, à la suite de Merleau-Ponty ${ }^{10}$ plutôt que de Heidegger, nomme notre "être au monde", paradigmatisme qui cependant ne permettra pas à la technique d'être davantage, dans la "réalité humaine», qu'une "phase " procédant du « déphasage » de l'« unité magique » en technique et religion.

11 Or, les tensions théoriques ici à nouveau pressenties pourraient bien n'être que la traduction, dans la Thèse complémentaire, des tensions présentes au sein de la Thèse principale. Surtout, l'idée de l'objet technique comme support de la relation transindividuelle doit pour mon propos être revalorisée, car c'est elle qui permettra de résoudre la difficulté ultime dont il s'agit maintenant de révéler la présence au sein de la Thèse principale. Le texte sans doute le plus profond et problématique à la fois sur le transindividuel est en effet celui consacré à la "problématique de la réflexivité dans l'individuation ", dans lequel on trouve le passage suivant :

En fait, ni l'idée d'immanence ni l'idée de transcendance ne peuvent rendre compte complètement des caractères $d u$ transindividuel par rapport à l'individu psychologique; la transcendance ou l'immanence sont en effet définies et fixées avant le moment où l'individu devient un des termes de la relation dans laquelle il s'intègre, mais dont l'autre terme était déjà donné. Or, si l'on admet que le transindividuel est auto-constitutif, on verra que le schème de transcendance ou le schème d'immanence ne rendent compte de cette auto-constitution que par leur position simultanée et réciproque; c'est en effet à chaque instant de l'autoconstitution que le rapport entre l'individu et le transindividuel se définit comme ce qui DÉPASSE L'INDIVIDU TOUT EN LE PROLONGEANT : le transindividuel n'est pas extérieur à l'individu et pourtant se détache dans une certaine mesure de l'individu ${ }^{11}$.

12 Afin de problématiser ce passage, je rappelle d'abord ce qui était apparu dans l'enquête du dernier chapitre de mon Penser l'individuation: chez Simondon le dédoublement psycho-somatique du vivant fabrique la "voie transitoire » psychique qui concerne le "sujet», dont la «personnalité » est, après "désindividuation émotionnelle provisoire ", l'actualisation transindividuelle, lieu paradoxal de l'individualité la plus grande comme à la fois de la subversion la plus accomplie de l'opposition individu/milieu - le social n'étant même plus un milieu. C'est pourquoi le transindividuel ou « collectif réel » est la forme actualisée du psychisme lui-même: «L'individualité psychologique 
apparaît comme étant ce qui s'élabore en élaborant la transindividualité12 ${ }^{12}$. Or, Simondon précise que cette subversion par le transindividuel de l'opposition entre immanence et transcendance tient à ce qu'«il y a une antériorité du transindividuel par rapport à l'individuel », cette antériorité étant en effet ce qui « empêche de définir un rapport de transcendance ou d'immanence ${ }^{13} »$. Mais une telle antériorité ne peut signifier qu'il y aurait équivalence entre le transindividuel et le préindividuel, même si certains passages favorisent la confusion, comme c'est le cas de ces lignes : « Le psychosocial est du transindividuel : c'est cette réalité que l'être individué transporte avec lui, cette charge d'être pour des individuations futures ». Cette confusion possible n'est que l'autre aspect d'une insistance - cruciale, elle - sur le fait que l'individuation transindividuelle - car c'en est bien une - construit l'individualité radicale au-delà même de l'individu, parce que c'est le «sujet» comme ensemble individu-préindividuel qui s'individue. C'est là ce qui rend l'individuation transindividuelle difficilement pensable, sinon en disant avec Simondon, dans le passage cité plus haut, que «le transindividuel se définit comme ce qui DÉPASSE L'INDIVIDU TOUT EN LE PROLONGEANT : le transindividuel n'est pas extérieur à l'individu et pourtant se détache dans une certaine mesure de l'individu ».

13 Reste que la difficulté représentée par l'idée d'antériorité du transindividuel n'est pas par là résolue. Si l'antériorité du transindividuel sur l'individuel ne signifie pas qu'il y aurait équivalence entre le transindividuel et le préindividuel, comment dès lors lui donner sens? Or, c'est ici que l'idée de l'objet technique comme support du transindividuel me semble pouvoir opérer. Car ce support est d'abord le "symbole » qui « exprime », comme disait Simondon, la part préindividuelle rattachée au "sujet». Dès lors, passer de l'idée de l'objet technique comme "symbole " à celle de l'objet technique comme "support", c'est concevoir que l'objet technique recevant la part préindividuelle du « sujet » est aussi et réciproquement ce qui fait accéder ce « sujet » à l'individuation transindividuelle dans sa distinction d'avec le préindividuel. L'objet technique serait alors cette médiation par laquelle le transindividuel se constitue dans son incompréhensible indissociabilité psycho-sociale, parce qu'il offrirait le lieu recherché par Simondon dans la Thèse principale sous le nom de ce qui «intériorise l'extérieur » et " extériorise l'intérieur ${ }^{14}$ », et qui comme tel est " antérieur ».

Mais Simondon pense la technique au-delà de l'artefact simple, sous prétexte que la technique ne se "concrétise » vraiment que dans la machine moderne, et il n'ira ainsi jamais jusqu'à poser l'objet technique à la fois comme « expression » du préindividuel rattaché au "sujet " et comme fondement de l'individuation transindividuelle, écrivant au contraire qu'«entre homme et nature se crée un milieu technogéographique qui ne devient possible que par l'intelligence de l'homme: l'autoconditionnement d'un schème par le résultat de son fonctionnement nécessite l'emploi d'une fonction inventive d'anticipation qui ne se trouve ni dans la nature ni dans les objets techniques déjà constitués ${ }^{15}$ ». S'il faut donc reconnaître ici mon « infidélité » à la lettre du texte de Simondon, la question se pose tout au moins de savoir si le transindividuel, tel que Simondon lui-même tente de le penser à travers sa Thèse principale puis sa Thèse complémentaire - c'est-à-dire tel qu'il s'en embarrasse et s'en trouve plongé dans des tensions théoriques - n'est pas en fait artefactuellement fondé. Dans une telle perspective, on pourrait dire avec Stiegler que la finitude du vivant oblige ce dernier à ne pouvoir s'individuer transindividuellement, donc en " personnalité » psycho-sociale 
pour parler avec Simondon, qu'en s'appuyant sur ces « béquilles de l'esprit » que sont les artefacts non-vivants.

\section{Les deux sens de la mort et l'apoptose comme « mort vitale »}

J'en viens maintenant à ce vivant lui-même avant son individuation psycho-sociale, pour montrer en quoi il est lui aussi rendu possible par une forme de «non-vie » : la vie du vivant ne se développe qu'en passant par l'apoptose ou "suicide cellulaire». Simondon lui-même avait, dans un passage de L'individu et sa genèse physico-biologique, dédoublé l'idée de mort afin de penser une certaine constitutivité de la mort par rapport à la vie :

la mort existe pour le vivant en deux sens qui ne coïncident pas : elle est la mort adverse [...]. Mais la mort existe aussi pour l'individu en un autre sens : l'individu n'est pas pure intériorité: il s'alourdit lui-même du poids des résidus de ses opérations ; il est passif par lui-même ; il est à lui-même sa propre extériorité [...]. En ce sens, le fait que l'individu n'est pas éternel paraît ne pas devoir être considéré comme accidentel ; la vie dans son ensemble peut être considérée comme une série transductive; la mort comme événement final n'est que la consommation d'un processus d'amortissement qui est contemporain de chaque opération vitale en tant qu'opération d'individuation; toute opération d'individuation dépose de la mort dans l'être individué qui se charge ainsi progressivement de quelque chose qu'il ne peut éliminer ; cet amortissement est différent de la dégradation des organes ; il est essentiel à l'activité d'individuation ${ }^{16}$.

Parce que la mort, entendue au second sens, n'est ici qu'un dépôt de l'individuation vitale, elle pourrait sembler se confondre avec la mort entendue au premier sens. En effet l'idée d'un dépôt, même nécessaire plutôt qu'accidentel, ne permet pas encore de penser une constitutivité du mort par rapport au vivant. Car le dépôt, comme tel, est "dépourvu de potentiels et ne peut plus être la base de nouvelles individuations ${ }^{17}$ ". Mais la différence réside en ce que la mort au premier sens « traduit la précarité même de l'individuation, son affrontement aux conditions du monde ", tandis que la mort au second sens "ne provient pas de l'affrontement au monde, mais de la convergence des transformations internes ${ }^{18} »$. Loin de moi l'idée que Simondon aurait pensé l'apoptose comme condition de la vie à une époque où la biologie en était encore à s'interroger sur la nature de l'apoptose. Simplement, il entre dans la logique d'une pensée de l'individuation de vouloir, comme l'a en effet voulu Simondon, subvertir toutes les oppositions classiques et même celle entre la vie et la mort, pour peu qu'on distingue entre des échelles d'individuation.

Or, la biologie actuelle est en mesure d'affirmer, comme le fait Jean-Claude Ameisen dans son ouvrage La sculpture du vivant, que la mort est au cœur même de la vie. L'ouvrage de Jean-Claude Ameisen me semble en fait révéler deux aspects différents de cette présence. D'une part, la construction de l'embryon implique l'auto-destruction d'un grand nombre de cellules. D'où les métaphores de la "sculpture» et de sa condition le «suicide » cellulaire, appliquées non seulement à la formation du cerveau et du système immunitaire, mais aussi à celle de l'organisme dans son ensemble :

Dès les premiers jours qui suivent notre conception - au moment même où débute notre existence - le suicide cellulaire joue un rôle essentiel dans notre corps en train de se construire, sculptant les métamorphoses successives de notre forme en devenir. Dans les dialogues qui s'établissent entre les différentes familles de cellules 
en train de naître, le langage détermine la vie ou la mort. Dans les ébauches de notre cerveau et de notre système immunitaire - l'organe qui nous protégera des microbes - la mort cellulaire est partie intégrante d'un processus étrange d'apprentissage et d'auto-organisation dont l'aboutissement n'est pas la sculpture d'une forme mais celle de notre mémoire et de notre identité. [...] C'est la mort cellulaire qui, par vagues successives, sculpte nos bras et nos jambes à partir de leurs ébauches, à mesure qu'elles grandissent, de leur base vers leur extrémité. À l'intérieur de nos avant-bras, elle crée l'espace qui sépare nos os, le radius et le cubitus. Puis elle sculpte les extrémités de nos membres: notre main naît tout d'abord sous la forme d'une moufle, d'une palme, contenant cinq branches de cartilage qui se projettent à partir du poignet et préfigurent nos doigts. La mort fait alors brutalement disparaître les tissus qui joignaient la portion supérieure de ces branches, individualisant nos doigts et transformant la moufle en gant ${ }^{19}$.

D'autre part, et ce second aspect vérifie en même temps que le premier aspect est bien une auto-destruction des cellules, toute cellule est équipée à la fois pour s'auto-détruire et pour empêcher cette auto-destruction, de sorte que la vie de l'organisme une fois formé n'est qu'une mort empêchée, et qui ne l'est d'ailleurs pas longtemps pour toutes les cellules qui doivent être chaque jour ou presque renouvelées, comme les cellules de la peau :

Quelle que soit leur durée de vie normale dans nos corps, de quarante-huit heures à quelques semaines, de quelques mois à plusieurs années, de quelques dizaines d'années à peut-être plus d'un siècle, chacune des cellules qui nous composent est, en permanence, à chaque instant, capable de s'auto-détruire. Et elle déclenchera en quelques heures - au plus en quelques jours - son suicide, si elle est privée des signaux qui lui permettent de survivre. Au début des années 1990, une nouvelle notion de la vie émergea : vivre, pour chaque cellule qui compose notre corps, c'est, à chaque instant, avoir réussi à réprimer le déclenchement de son suicide. La différenciation qui conduit, dans les différentes familles cellulaires, au verrouillage de la plupart des gènes $-\mathrm{y}$ compris, dans de nombreuses familles cellulaires, dont les neurones, au verrouillage des gènes qui permettent aux cellules de se dédoubler - n'oblitère jamais, semble-t-il, dans aucune cellule, tout au long de notre vie, certaines des informations génétiques permettant de déclencher l'exécution du suicide. [...] Le suicide quotidien de centaines de milliards de cellules dans notre corps ne représente que la manifestation visible d'une potentialité permanente, ancrée dans chacune de nos cellules ${ }^{20}$.

\section{Conclusion : de la vie comme différence à soi ou « non-essence »}

19 Au terme de ce rapide examen des types de «non-vie » qui conditionnent la vie comme évolution biologique puis comme histoire psycho-sociale, on peut faire une hypothèse sur la nature de ce que l'on nomme ainsi la « vie » : cette «nature » de la vie est peutêtre justement une anti-nature ou une "non-essence ", parce que la vie se définirait comme différence à soi si :

- elle s'ancre dans ce qui n'est pas elle (le non-vivant chimique);

- elle évolue en utilisant la mort comme potentialité inscrite en chaque cellule ;

- elle est capable de se sublimer en une vie psycho-sociale où s'accomplit pleinement son caractère de non-essence puisque l'homme, dont on dit qu'il est historique et n'a pas de «nature », construit son esprit et sa personnalité par un processus d'« extériorisation » dans des artefacts qui paradoxalement conditionnent le développement de son « intériorité ». 


\section{BIBLIOGRAPHIE}

Ameisen Jean-Claude, La Sculpture du Vivant. Le Suicide cellulaire ou la Mort créatrice, Paris, Seuil, 2003.

Barthélémy Jean-Hugues, Penser l'individuation. Simondon et la philosophie de la nature (préface de Jean-Claude Beaune), Paris, L'Harmattan, 2005.

Colson Daniel, Petit lexique philosophique de l'anarchisme, de Proudhon à Deleuze, Paris, Le Livre de Poche, 2003.

Guchet Xavier, «Théorie du lien social, technologie et philosophie : Simondon lecteur de Merleau-Ponty ", Les études philosophiques, $\mathrm{n}^{\circ}$ 2, Paris, 2001.

Marx Karl, Le Capital, Livre 1, Joseph Roy (trad.), Paris, Garnier-Flammarion, 1969.

Simondon Gilbert, Du mode d'existence des objets techniques, Paris, Aubier, 1958.

Simondon Gilbert, L'individuation psychique et collective, Paris, Aubier, 1989.

Simondon Gilbert, L'individu et sa genèse physico-biologique, Grenoble, Millon, 1995.

Simondon Gilbert, L'individuation à la lumière des notions de forme et d'information, Grenoble, Millon, 2005.

Simondon Gilbert, Penser la connaissance et la technique après Simondon, Paris, L'Harmattan, 2005.

Simondon Gilbert, Simondon ou l'Encyclopédisme génétique, Paris, PUF, 2008.

\section{NOTES}

1. Gilbert Simondon, L'individuation à la lumière des notions de forme et d'information, Grenoble, Millon, 2005.

2. Jean-Hugues Barthélémy, Penser l'individuation. Simondon et la philosophie de la nature (préface de Jean-Claude Beaune), Paris, L'Harmattan, 2005.

3. Karl Marx, Le Capital, Livre 1, Joseph Roy (trad.), Paris, Garnier-Flammarion, 1969, p. 36.

4. Ibid. (souligné par l'auteur).

5. Gilbert Simondon, Du mode d'existence des objets techniques, Paris, Aubier, 1958. Voir également mon commentaire dans la deuxième partie de Penser la connaissance et la technique après Simondon, Paris, L'Harmattan, 2005, ainsi que plus récemment dans le dernier chapitre de mon ouvrage de synthèse Simondon ou l'Encyclopédisme génétique, Paris, PUF, 2008.

6. Voir Penser la connaissance et la technique après Simondon, Paris, L'Harmattan, 2005, deuxième partie, chapitre II, 4 .

7. Gilbert Simondon, Du mode d'existence des objets techniques, p. 247-248 (souligné par l'auteur).

8. Ibid., p. 248. La non-contradiction entre la critique simondonienne de l'anthropologie essentialiste et l'idée d'un support technique du transindividuel a été développée dans mon Penser la connaissance et la technique après Simondon, à l'occasion de l'exégèse polémique de Du mode d'existence des objets techniques.

9. Simondon y écrivait en effet: «L'opposition dressée entre la culture et la technique, entre l'homme et la machine, est fausse et sans fondement; elle ne recouvre qu'ignorance ou ressentiment. Elle masque derrière un facile humanisme une réalité riche en efforts humains et en forces naturelles, et qui constitue le monde des objets techniques, médiateurs entre la nature 
et l'homme" (Du mode d'existence des objets techniques, p. 9). Ce sont à vrai dire les trois oppositions mentionnées qui sont ici combattues d'un même geste. À l'«humanisme facile» Simondon substitue, non pas un technicisme - ni du reste un «naturalisme » - qui serait un antihumanisme, mais un humanisme difficile car pariant sur la subversion des oppositions croisées entre nature, humanité et technique. C'est pourquoi je ne peux adhérer à la présentation qu'en fait Daniel Colson dans son Petit lexique philosophique de l'anarchisme, de Proudhon à Deleuze (Paris, Le Livre de Poche, 2003). Du reste, si Deleuze fit la première recension - fort élogieuse - de L'individu et sa genèse physico-biologique et fut personnellement inspiré par cet ouvrage, c'est sur de tout autres points, relatifs à sa pensée de la «différence» et du «champ transcendantal impersonnel et préindividuel »- même si là aussi on pourrait dénoncer une récupération. Pour ce qui concerne l'anti-humanisme et l'anarchisme, Simondon s'en rapproche moins que de l'excellent Pour l'homme de son ami Mikel Dufrenne, dont les critiques subtiles - adressées à l'anti-humanisme - seront au service de cet "humanisme difficile» qui correspond à la subversion, prioritaire chez un phénoménologue comme Dufrenne, des alternatives classiques. Sur cette question, voir mon Simondon ou l'Encyclopédisme génétique.

10. Sur une certaine anticipation, par le Merleau-Ponty de Signes notamment, de la pensée simondonienne de la technique, voir Xavier Guchet, "Théorie du lien social, technologie et philosophie : Simondon lecteur de Merleau-Ponty ", Les études philosophiques, n² 2, Paris, 2001.

11. Gilbert Simondon, L'individuation psychique et collective, Paris, Aubier, 1989, p. 156 (souligné par l'auteur). Rappelons que cet ouvrage de Simondon livre le dernier tiers de sa Thèse principale, désormais parue de manière unifiée et complète sous le titre L'individuation à la lumière des notions de forme et d'information.

12. Ibid., p. 157.

13. Ibid., p. 195.

14. Ibid., p. 157.

15. Gilbert Simondon, Du mode d'existence des objets techniques, p. 57 (souligné par l'auteur).

16. L'individu et sa genèse physico-biologique, Grenoble, Millon, 1995, p. 213-214. Rappelons que cet ouvrage de Simondon livre les deux premiers tiers de la Thèse principale. Quant au passage cité, sans doute n'est-ce pas un hasard si Simondon l'écrit lorsqu'il anticipe une seconde fois - après cette première anticipation qu'était le sous-chapitre «les niveaux successifs d'individuation »sur le traitement du « collectif » dans son rapport à « l'individuation du vivant ».

17. Ibid.

18. Ibid., p. 213 (nous soulignons).

19. Jean-Claude Ameisen, La Sculpture du Vivant. Le Suicide cellulaire ou la Mort créatrice, Paris, Seuil, 2003, p. 16 et 40 .

20. Ibid., p. 138.

INDEX

Personnes citées : Simondon (Gilbert)

Mots-clés : apoptose, artefact, mort, transindividuel, vie 


\section{AUTEUR}

\section{JEAN-HUGUES BARTHÉLÉMY}

Jean-Hugues Barthélémy est docteur en épistémologie et histoire des sciences et techniques (université Paris 7 - Denis Diderot). Il a dirigé le séminaire « Simondon ou l'Encyclopédisme génétique » de la Maison des sciences de l'Homme de Paris-Nord entre 2006 et 2008. Il est l'auteur de trois ouvrages sur Simondon, dont le dernier est paru en mai 2008 aux PUF : Simondon ou l'Encyclopédisme génétique. 